\title{
Clinicopathological, immunohistochemical and treatment features of metachronous versus synchronous bilateral breast carcinoma
}

\author{
Oana-Adriana RAJPUT-ANGHEL ${ }^{1}$, Traean BURCOS ${ }^{1,2}$ \\ ${ }^{1}$ Department of General Surgery, Coltea Clinical Hospital, Bucharest, Romania \\ 2"Carol Davila" University, Department Of General Surgery, Bucharest, Romania
}

\begin{abstract}
Objectives. The purpose of this study was to assess and compare the clinicopathological, molecular pathology, treatment and survival characteristics in patients with metachronous bilateral breast cancer $(m B B C)$ and synchronous breast cancer ( $s B B C)$

Materials and methods. A cohort of 658 patients with breast cancer treated at the Coltea Clinical Hospital, Surgery Department, between January 2015 and December 2019 and followed-up until August 2020 was studied. Data pertaining to patients who were diagnosed as having bilateral breast cancer were retrospectively reviewed and collected. A 3-months interval was used to distinguish metachronous from synchronous tumors. Among patients with bilateral breast cancer, assessment parameters included patient characteristics, histological and molecular pathology features and the performed treatment that were statistically evaluated comparing the first and second tumor of each group and among groups. Survival analysis was performed comparing $m B B C$ and $s B B C$ patients. SPSS was used for data analysis.

Outcomes. Of the 658 patients with primary breast cancer, 35 (5.3\%) patients were diagnosed as having bilateral breast cancer $(25(3.8 \%) \mathrm{mBBC}$ and 10 (1.5\%) sBBC). When clinical and histopathological parameters were statistically evaluated, age, menopausal status, tumor size, number of invaded nodes and anatomic stage were found to be significant between the tumors of the metachronous group and tumor size, pathologic T(tumor) and stage between tumors of the synchronous group. Hormonal receptor (HR) status concordance was higher in the synchronous group $(85.7 \%, p=0.010)$, with a higher percentage of ER positive $(71.4 \%)$ and PR positive $(71.4 \%)$ concordance of the tumors. In terms of survival analysis, there was a difference in overall survival (OS, $p=0.005)$, disease-free survival (DFS, $p=0.011)$ and distant relapse-free survival $(p=0.003)$ between $m B B C$ and $s B B C$. The mean disease-free survival for patients in whom metachronous tumor occurred within less than 5 years was 63.3 months, for sBBC patients was 39.6 months, whereas for patients with more than 5 years was 437.9 months ( $p=0.012$, Log Rank). Discordant biomarker defined subgroup (ER,HER2) patients were associated with better disease-free survival ( $p=$ 0.047, Log Rank) and better distant relapse-free survival ( $p=0.015$, Log Rank) in overall patients. In terms of loco-regional relapse-free survival, although $m B B C$ and $s B B C$ patients showed no statistical significant difference earlier in the time course ( $p=0.088$, Breslow; $p=0.054$ Tarone-Ware), among $m B B C$ patients was observed a better outcome ( $p=0.027$, Log Rank).

Conclusions. Based on survival analysis, patients in whom metachronous tumor developed after more than 5 years, had a better distant relapse-free survival. Patients with synchronous bilateral breast cancer were associated with worse disease outcome based on overall survival analysis and disease free-survival
\end{abstract}


rates with more frequent rates of distant metastasis. Outcome of patients in whom metachronous tumor was diagnosed within less than 5 years might be similar to synchronous tumors. Patients with discordant ER,HER2 status showed a better disease outcome. Although concordance in HR status and molecular subtype, did not show statistical significant differences, it is a subject which deserves further clinical observation.

Keywords: bilateral breast cancer, synchronous, metachronous, survival, local-regional relapse, distant relapse

\section{INTRODUCTION}

According to International Agency for Research on cancerce (IARC), GLOBOCAN 2020 estimates of cancer incidence and mortality, breast cancer has surpassed lung cancer and is the most commonly diagnosed malignancy in women (24.5\%) and both males and females (11.7\%) (with an estimated number of 2,261,419 new cases) and is the fifth cause of cancer death $(6.9 \%$, 684,996 estimated deaths) worldwide (1). In Romania, according to the most recent report of the regional tumor registry and IARC, breast cancer was the most frequent cancer in women with $26.9 \%$ of newly diagnosed cancer cases $(12,085$ estimated new cases, $65.8 / 100,000$ incidence) and also the first in terms of specific mortality with an estimated 3918 number of deaths in 2020 (17.3\%, 17.4/100,000 mortality rate) (2$6)$.

Based on the interval time between the diagnosis of first and the second tumor, bilateral breast cancer (BBC) can be classified into metachronous ( $\mathrm{mBBC}$ ) and synchronous ( $\mathrm{SBBC})(5,6)$. According to the World Health Organization (WHO), BBC is defined as SBBC when contralateral breast carcinoma is diagnosed at the same time as the first tumor or within 3 months. When contralateral tumor is diagnosed three months or more after the first tumor, is defined as $\mathrm{mBBC}$. (510). Some authors have defined $\triangle B B C$ as the diagnosis of the second within 12 months $(6-8,11)$.

As a result of constantly increasing incidence of breast cancer, improved diagnosis methods, progress in systemic and loco-regional treatment and longer life expectancy, the incidence of BBC has been increasing $(5,12-15)$. Reported studies frequency of bilateral breast cancer varies from $1.4 \%$ to $11.8 \%$. $(5,9,12,15$ 17). The risk of developing a second primary breast cancer in women who have had previous breast cancer is predicted as 2 to 6 times greater than the first breast cancer in the general population $(12,18)$.

Studies results are still controversial regarding the impact of contralateral breast cancer on survival and still remains uncertain $(5,6,9,19-22)$. Some studies have shown that it remains unaffected while on the contrary, others have suggested that the development of cancer in the contralateral breast negatively affects survival, beeing associated with worse outcome and reduced survival $(12,14,19,23,24)$.

Up to this point there is no specific recommendations for the treatment of bilateral breast cancer $(5,9,19,21-23)$. For synchronous breast cancer, systemic treatment is usually guided by the SBBC tumor with the worse prognosis, whereas metachronous breast cancer tend to be treated like unilateral, independent breast cancer (5).

With these means, the present study aimed to assess patients diagnosed as having bilateral breast cancer and to identify differences in clinical, pathological characteristics and type of performed surgery of the first and second tumor and to compare overall survival rates and disease-free periods.

\section{MATERIAL AND METHODS}

Data pertaining to patients diagnosed as having bilateral breast cancer, who were treated for at least one tumor from January 2015 to December 2019 and followed-up until August 2020 at the Coltea Clinical Hospital Bucharest, General Surgery Department, were reviewed retrospectively. Data were gathered from the medical files of the institution (Hospital Manager Suite, InfoWorld; pathologic reports).

Based on the time interval of the diagnosis of cancer in the contralateral breast, patients were divided in two groups: synchronous bilateral breast cancer (sBBC) group and metachronous bilateral breast cancer ( $\mathrm{mBBC}$ ) group. According to the WHO classification of bilateral breast cancer, contralateral breast cancers detected within 3 months from the diagnosis of first tumor were defined as synchronous breast cancer. Regarding the sBBC group, the tumor with larger diameter was considered the first tumor and identified as the dominant tumor. Any second tumor diagnosed in contralateral breast after 3 months was considered metachronous breast cancer.

Inclusion criteria were patients diagnosed with bilateral breast cancer, confirmed pathologically with invasive breast cancer and those who underwent surgery. Patients with the one side tumor described from the pathologists as likely being a metastatis from the contralateral breast, were excluded. 
Family history of the patients (considered positive when a first or second degree relative had breast cancer), genetic mutations, tobacco smoking, alcohol consumption, age at first and second tumor, the time elapsed between development of two tumors, state of menopause, the type of performed surgery, axillary dissection and systemic treatment were recorded. From the pathology reports were extracted the diameter of the tumor (in $\mathrm{mm}$ ), number of tumor foci and tumor focality, histological type, histologic grade (Scarff-Bloom-Richardson - SBR grading system, Nottingham modification), associated ductal or lobular carcinoma in situ (DCIS/LCIS), lymphovascular invasion (LVI), perineural invasion (PNI), status of resection margins, lymph node status, anatomical pathological stage (TNM), estrogen receptor (ER), progesterone receptor (PR) and human epidermal growth factor receptor 2 (HER2) status and Ki-67 proliferation marker index value.

The performed surgical procedures were classified into three categories: breast-conserving surgery, subcutaneous mastectomy and mastectomy with none, partial or radical axillary lymphadenectomy.

Tumors $(T)$ were classified as smaller than or equal to $20 \mathrm{~mm}$ (T1), >20 $\leq 50 \mathrm{~mm}$ (T2), larger than $50 \mathrm{~mm}$ (T3) and T4 (direct extension to the chest wall and/or to the skin) as described in AJCC (American Joint Committee on Cancer) Staging Manual Eighth Edition, 2018. In terms of histological features, types were classified according to the WHO Classification of Tumors, fifth edition, 2019, as (a) invasive breast carcinoma of no special type (IBC-NST) (respectively invasive ductal carcinoma (IDC), (b) invasive lobular carcinoma (ILC), (c) mixed IBC-NST and special subtypes, (d) IBC-NST with focal specialized subtype and (e) other special subtypes; if the special subtype carcinoma made up 10$90 \%$ of the cancer, was used the term „mixed IBC-NST and special subtype carcinoma”, whereas cancers with $<10 \%$ special subtype were classified as „IBC-NST with focal specialized subtype".

Histological grades were assigned from well differentiated grade (grade 1, G1), moderately differentiated grade (grade 2, G2) to poorly differentiated grade (grade 3, G3).

Axillary node involvement was categorized as negative (N0) or positive N1(metastase in 1-3 axillary lymph nodes), N2(4-9 axillary lymph nodes) and N3 (ten or more axillary lymph nodes) (AJCC Cancer Staging Manual, 8th ed).

Estrogen, progesterone and HER2 receptors were evaluated using immunohistochemical methods with in situ hybridization (ISH, chromogenic and or fluorescence in situ hybridization (CISH, FISH)) where appropriate. ER and PR positivity were defined as no less than $1 \%$ stained nuclei according to the American Society of Clinical Oncology/College of American Patholo- gists (ASCO/CAP) Practice Guideline Update recommendations. Invasive cancers with 1-10\% ER positivity were reported as ER low positive. HER2 status was considered positive with a $3+$ stain intensity expression and negative when absent or with 1+ expression. In situ hybridization classified further HER2 status in positive or negative for equivocal $2+$ tumors. Based on measurements of ER, PR, HER2 and Ki-67, invasive breast carcinomas were further classified into the five molecular subtypes, adopted by the 13th St. Gallen International Breast Cancer Conference (2013) Expert Panel: (1) Luminal A, (2) Luminal B-like HER2 negative, (3) Luminal B-like HER2 positive, (4) HER2-positive (non-luminal) and (5) Triple-negative breast cancer (TNBC). A threshold of $14 \%$ Ki-67 value was used to discriminate between less agressive behaviour Luminal $A$ molecular subtype and more agressive Luminal B subtype. As published in fifth edition of the WHO Classification of Breast Tumors, 2019 (WHO Blue Books), tumors were also divided into the following biomarker defined subtypes: (1) ER-positive, HER2-negative; (2) ER-positive, HER2-positive; (3) ER-negative, HER2-positive; (4) ER-negative, HER2 negative. Based on the hormone receptor (HR) we had the following groups (1) $\mathrm{ER}+, \mathrm{PR}-$; (2) ER+.PR+; (3) ER-,PR+; (4) ER-,PR-.

Loco-regional recurrence (breast, axilla or both relapse) and distant metastasis were also recorded. Distant metastases were defined as any lesion located outside the mastectomy scar or restant breast tissue, axillary or regional (supraclavicular) lymph nodes. Metastatic sites were grouped as single or multiple, subsequently into M1 bone/lung, M1 bone+lung/brain/ lung+liver/lung+ distant lymph nodes.

Overall survival (OS) (was calculated from the date of diagnosis of the first tumor until the date of death or the last medical assessement), disease-free survival (DFS, calculated until the date of relapse), loco-regional relapse-free survival (RFS) and distant relapse-free survival rates were calculated with censoring for loss of follow-up. All the mentioned variables were compared between groups.

\section{Statistical analysis}

Statistical analyses were performed using IBM SPSS Statistics software version 28.0.0.0 (190). The variables were investigated using descriptive statistics (Frequencies and Descriptives) and presented using median, mean and standard deviation for normally distributed variables. Normality tests Kolmogorov-Smirnov and Shapiro-Wilk were used to determine whether variables were normally distributed. Non-parametric categorical variables were investigated using the Mann-Whitney U test, Pearson Chi-square test or Fisher's exact test, after exclusion of missing values. Survival outcomes were estimated using Kaplan-Meier sur- 
vival analysis and compared by Log Rank, Breslow and Tarone-Ware tests. For all statistical analyses, a $p$ value of less than or equal to 0.05 was defined as statistically significant.

\section{OUTCOMES}

Of the 658 patients who were diagnosed as breast cancer and treated for invasive breast carcinoma between January 2015 and December 2019, a total of 35 (5.3\%) patients were identified and diagnosed as having bilateral breast cancer (BBC). Seven of the cases were males and all of them were unilateral. A number of $10(1.5 \%)$ of the 35 bilateral breast cancer cases had synchronous tumor (28.6\%). 25 (3.8\%) of the 35 BBC had metachronous breast cancer (71.4\%) ( $p=.011 \mathrm{Chi}$ Square test). The mean time interval between two tumors was 0 months in sBBC group and 112.80 months (range 16-372 months) in $\mathrm{mBBC}$ group (the median time interval was 66 months). The median age of sBBC patients was 59.50 years, while for $\mathrm{mBBC}$ group the median age at the diagnose of the first tumor was 58 years and 65 years for the second tumor and 18 (72\%) patients were in the postmenopausal period. The mean follow-up time for $\mathrm{mBBC}$ patients was 136.92 months (median 93.0, range 17-528 months), 19.60 months (median 14.50, range 1-60 months) for sBBC and 103.40 (median 51 months) for both groups $(p=<001)$.

Patient characteristics, the performed surgical treatment and histological features (descriptives, frequencies, statistical values) regarding metachronous and synchronous tumors are summarized individually for each group in Tables 1 and 2. At inclusion, patients did not show any differences between groups except for one case (4\%) that harboured BRCA1 genetic mutation, which was found in $\mathrm{mBBC}$ group. None of the groups have had family history of breast cancer and only two patients (20\%) acknowledged smoking in the sBBC group. With regard to metachronous tumors, there was found a statistically significant difference between first and second tumor in terms of age $(p<0.001)$ and menopausal status ( $p<0.001)$, all patients beeing menopaused by the time the second tumor was diagnosed. Concerning the comparison of metachronous and synchronous tumors, there were no significant differences regarding the age at intial diagnosis $(p=0.339)$ and menopausal status ( $p=0.928$ ).

The type of performed surgery did not differ among groups ( $p=0.255,0.174$ ) or between first and second tumors ( $p=0.531,0.684)$. Mastectomy was the most common surgical intervention performed to both breasts. Radical axillary lymphadenectomy was performed in all patients in the synchronous group, al- though no statistically significant difference between groups or tumors $(p=0.733)$.

Regarding tumor pathology features, no statistically significant difference was observed between the histological types of the first and second tumors for $\mathrm{mBBC}$ and $\mathrm{sBBC}(\mathrm{p}=0.631)$. In both groups, the most common histologic type was invasive ductal carcinoma-NOS ( $p=0.313)$. Histological type concordance was $28.6 \%$ ( $p=0.109$ ) for $m B B C$ group and $50 \%(p=1.000)$ for sBBC group. Histological grade concordance was $27.3 \%$ $(p=0.132)$ for $m B B C$ patiens and $37.5 \%(p=0.480)$ for sBBC patients. Regading histological type and grade between the two groups, the difference was not significant ( $p=0.286, p=0.637)$.

For metachronous group, the mean tumor diameter was $43.85 \mathrm{~mm}$ in the first tumor and $21 \mathrm{~mm}$ in the second tumor $(p=0.004)$. Concerning synchronous tumors the mean tumor size in the 1 st was $33.40 \mathrm{~mm}$ and $17.40 \mathrm{~mm}$ in the $2 \mathrm{nd}$ ( $p=0.011$ ). Second tumors for both $\mathrm{mBBC}$ and $\mathrm{sBBC}$ were more often of small size $(<20 \mathrm{~mm})(p=0.023, \mathrm{mBBC} ; \mathrm{p}=0.035 \mathrm{sBBC})$. The mean tumor diameter was therefore significantly greater in the dominant breast, with no statistically significant difference between groups ( $p=0.784)$. No differences were observed in terms of focality, histological grade, intraductal component, lymphovascular or perineural invasion. In synchronous group, pathologic T1 was more often encountered, in $70 \%$ of the cases, with no T4 in both tumors of the group ( $p=0.035)$. In terms of lymph node metastases, in the metachronous group, the second tumor was in $72 \%$ of the cases diagnosed with no regional lymph node invasion ( $p=0.026)$. Regarding anatomic pathologic stage TNM, in both metachronous and synchronous groups, when comparing the first tumor to the second, was found that stage IA had the highest incidence among second tumors, 56\% in $\mathrm{mBBC}$ group $(p=0.005)$ and $40 \%$ in $\mathrm{sBBC}(\mathrm{p}=0.007)$.

Features of the molecular pathology report are shown in Table 3. There was no statistically significant difference between the two tumors of the groups in terms of estrogen, progesterone and HER2 receptors. Estrogen receptor was negative in $48 \%$ of the cases ( $p=$ 0.370 ), in the first tumor of $\mathrm{mBBC}$ group, and, considering all combinations of estrogen and progesteron receptor expression and molecular subtype, ER-, PR-type (48\%, $p=0.387)$, ER-,HER2- (44\%, $p=0.217)$ and TNBC $(44 \%, p=0.111)$ were more often observed compared to the second tumor.

When comparing the first tumor of metachronous group with the dominant tumor of synchronous group, the distribution of the molecular subtypes differed among groups, in the $\mathrm{MBBC}$ group TNBC was more often diagnosed (44\%), whereas in the $\mathrm{SBBC}$ was Luminal B-like (HER2-negativ) (50\%) ( $p=0.035)$.

Concordance of biomarkers and intrinsec molecular subtypes between the first tumor and second tumor 
TABLE 1. Patient characteristics, surgical treatment and tumor histological features in metachronous tumors

\begin{tabular}{|c|c|c|c|}
\hline $\begin{array}{l}\text { Metachronous tumors, } n=25(100) \\
\text { Characteristics }\end{array}$ & $\begin{array}{l}\text { First tumor } \\
\quad n(\%)\end{array}$ & $\begin{array}{l}\text { Second tumor } \\
n(\%)\end{array}$ & $p$ value \\
\hline Median age, years (min-max), mean & $58(35-76), 55.56$ & $65(51-78), 65.40$ & $<0.001$ \\
\hline$\leq 40$ & $3(12)$ & $0(0)$ & \\
\hline $41-50$ & $4(16)$ & $0(0)$ & \\
\hline $51-60$ & $12(48)$ & $7(28)$ & \\
\hline$>60$ & $6(24)$ & $18(72)$ & \\
\hline Menopausal status & & & $<0.001$ \\
\hline Premenopausal & $7(28)$ & $0(0)$ & \\
\hline Postmenopausal & $18(72)$ & $25(100)$ & \\
\hline BRCA1 & $1(4)$ & & \\
\hline Breast surgery, type & & & 0.531 \\
\hline Breast conserving surgery & $1(4)$ & $2(8)$ & \\
\hline Subcutaneous mastectomy & $0(0)$ & $0(0)$ & \\
\hline Mastectomy & $24(96)$ & $22(88)$ & \\
\hline Unknown & $\mathrm{x}$ & $1(4)$ & \\
\hline Axillary surgery & & & 0.944 \\
\hline Partial axillar lymphadenectomy & $2(8)$ & $3(12)$ & \\
\hline Radical axillar lymphadenectomy & $23(92)$ & $20(80)$ & \\
\hline None & $0(0)$ & $1(4)$ & \\
\hline Unknown & $x$ & $1(4)$ & \\
\hline Tumor size $(\mathrm{mm})$, mean value $\pm \mathrm{SD}$; median; min- $\max$ & $\begin{array}{c}43.85 \pm 34.5 ; 30 \\
15-130 \\
\end{array}$ & $\begin{array}{l}21 \pm 12.84 ; 15 \\
6-60\end{array}$ & 0.004 \\
\hline Tumor size $(\mathrm{mm})$ groups & & & 0.023 \\
\hline$\leq 20 \mathrm{~mm}$ & $4(16)$ & $16(64)$ & \\
\hline$>20 \leq 50 \mathrm{~mm}$ & $10(40)$ & $7(28)$ & \\
\hline$>50 \mathrm{~mm}$ & $2(8)$ & $1(4)$ & \\
\hline Unknown & $9(36)$ & $1(4)$ & \\
\hline Tumor focality & & & 0.917 \\
\hline Unifocal & $12(48)$ & $21(84)$ & \\
\hline Multifocal & $1(4)$ & $2(8)$ & \\
\hline Multicentric & $1(4)$ & $1(4)$ & \\
\hline Unknown & $11(44)$ & $1(4)$ & \\
\hline Number of tumori foci & & & 0.940 \\
\hline Single focus & $12(48)$ & $21(84)$ & \\
\hline More than one focus & $2(8)$ & $3(12)$ & \\
\hline Unknown & $11(44)$ & $1(4)$ & \\
\hline Histological type & & & 0.627 \\
\hline \multicolumn{4}{|l|}{ 1.IBC-NST, ILC special subtype } \\
\hline IDC & $7(28)$ & $15(60)$ & \\
\hline ILC & $2(8)$ & $5(20)$ & \\
\hline mixed IDC + ILC & $x$ & $1(4)$ & \\
\hline \multicolumn{4}{|l|}{ 2.mixed IBC-NST and special subtypes } \\
\hline IDC + acinic cell carcinoma & $1(4)$ & $x$ & \\
\hline $\begin{array}{l}\text { IDC + metaplastic carcinoma (mixed, epithelial + } \\
\text { mesenchymal) }\end{array}$ & $x$ & $1(4)$ & \\
\hline \multicolumn{4}{|l|}{ 3.IBC-NST with focal specialized subtype } \\
\hline IDC + invasive papillary carcinoma & $1(4)$ & $1(4)$ & \\
\hline IDC + carcinoma with apocrine differentiation & $x$ & $x$ & \\
\hline
\end{tabular}




\begin{tabular}{|c|c|c|c|}
\hline $\begin{array}{l}\text { Metachronous tumors, } \mathrm{n}=25 \text { (100) } \\
\text { Characteristics }\end{array}$ & $\begin{array}{l}\text { First tumor } \\
\quad \mathrm{n}(\%)\end{array}$ & $\begin{array}{l}\text { Second tumor } \\
\mathrm{n}(\%)\end{array}$ & $\mathrm{p}$ value \\
\hline \multicolumn{4}{|l|}{ 4.Other special subtypes } \\
\hline Invasive papillary carcinoma & $1(4)$ & $x$ & \\
\hline Cribriform carcinoma & $1(4)$ & $\mathrm{x}$ & \\
\hline $\begin{array}{l}\text { Mucinous carcinoma with focal carcinoma with signet- } \\
\text { ring cell differentiation }\end{array}$ & $x$ & $1(4)$ & \\
\hline $\begin{array}{l}\text { Metaplastic carcinoma (with squamous differentiation) } \\
\text { with focal IDC }\end{array}$ & $x$ & $1(4)$ & \\
\hline Unknown & $12(48)$ & $x$ & \\
\hline Nottingham grade & & & 0.296 \\
\hline Grade 1 & $6(24)$ & $5(20)$ & \\
\hline Grade 2 & $5(20)$ & $14(56)$ & \\
\hline Grade 3 & $3(8)$ & $4(16)$ & \\
\hline Unknown & $12(48)$ & $2(8)$ & \\
\hline Associated DCIS/LCIS & & & 0.410 \\
\hline Low nuclear grade DCIS & $\mathrm{x}$ & $1(4)$ & \\
\hline Intermediate nuclear grade DCIS & $1(4)$ & $6(24)$ & \\
\hline High nuclear grade DCIS & $3(12)$ & $3(12)$ & \\
\hline LCIS & $1(4)$ & $1(4)$ & \\
\hline $\mathrm{DCIS}+\mathrm{LCIS}$ & $x$ & $1(4)$ & \\
\hline None & $9(36)$ & $12(48)$ & \\
\hline Unknown & $11(44)$ & $1(4)$ & \\
\hline Lymphovascular invasion & & & 0.940 \\
\hline Positive & $2(8)$ & $3(12)$ & \\
\hline Negative & $12(48)$ & $21(84)$ & \\
\hline Unknown & $11(44)$ & $1(4)$ & \\
\hline Perineural invasion & & & 0.560 \\
\hline Positive & $3(12)$ & $8(32)$ & \\
\hline Negative & $11(44)$ & $16(64)$ & \\
\hline Unknown & $11(44)$ & $1(4)$ & \\
\hline Margins & & & 0.327 \\
\hline RO & $24(96)$ & $24(96)$ & \\
\hline R1 & $1(4)$ & $0(0)$ & \\
\hline Unknown & $x$ & $1(4)$ & \\
\hline Pathologic T & & & 0.132 \\
\hline pT1 & $4(16)$ & $15(60)$ & \\
\hline pT2 & $8(32)$ & $5(20)$ & \\
\hline рT3 & $2(8)$ & $1(4)$ & \\
\hline pT4 & $2(8)$ & $4(16)$ & \\
\hline Unknown & $9(36)$ & $\mathrm{x}$ & \\
\hline Invaded lymph nodes, mean (min-max), SD & $6.94(0-45), 13.08$ & $1.48(0-25), 5.24$ & 0.026 \\
\hline Nodal status $(\mathrm{pN})$ & & & 0.051 \\
\hline $\mathrm{pNx}$ & $\mathrm{x}$ & $1(4)$ & \\
\hline pNO & $6(24)$ & $18(72)$ & \\
\hline $\mathrm{pN1}$ & $5(20)$ & $3(12)$ & \\
\hline $\mathrm{pN2}$ & $1(4)$ & $1(4)$ & \\
\hline pN3 & $4(16)$ & $1(4)$ & \\
\hline Unknown & $9(36)$ & $1(4)$ & \\
\hline Anatomic stage (TNM) & & & 0.005 \\
\hline Stage IA & $1(4)$ & $14(56)$ & \\
\hline Stage IB & $x$ & $x$ & \\
\hline Stage IIA & $7(28)$ & $3(12)$ & \\
\hline Stage IIB & $2(8)$ & $\mathrm{x}$ & \\
\hline Stage IIIA & $1(4)$ & $2(8)$ & \\
\hline
\end{tabular}




\begin{tabular}{|l|c|c|l|}
\hline $\begin{array}{l}\text { Metachronous tumors, } \mathbf{n}=\mathbf{2 5}(\mathbf{1 0 0}) \\
\text { Characteristics }\end{array}$ & $\begin{array}{c}\text { First tumor } \\
\mathbf{n}(\%)\end{array}$ & $\begin{array}{c}\text { Second tumor } \\
\mathbf{n}(\%)\end{array}$ & p value \\
\hline Stage IIIB & $1(4)$ & $1(4)$ & $1(4)$ \\
\hline Stage IIIC & $3(12)$ & $2(8)$ & \\
\hline Stage V & $1(4)$ & $2(8)$ & \\
\hline Unknown & $9(36)$ & & \\
\hline $\begin{array}{l}\text { Median time of metachronous cancer occurrence, } \\
\text { months (min-max), mean }\end{array}$ & $66(16-372), 112.80$ & & \\
\hline
\end{tabular}

$S D$ - standard deviation; IBC-NST - invasive breast carcinoma of no special type; IDC - invasive ductal carcinoma; ILC - invasive lobular carcinoma; DCIS - ductal carcinoma in situ; LCIS - lobular carcinoma in situ; RO - negative surgical margins; R1 - positive surgical margins

TABLE 2. Patient characteristics, surgical treatment and tumor histological features in synchronous tumors

\begin{tabular}{|c|c|c|c|}
\hline $\begin{array}{l}\text { Synchronous tumors, } n=10(100) \\
\text { Characteristics }\end{array}$ & $\begin{array}{c}\text { Dominant tumor } \\
\mathrm{n}(\%)\end{array}$ & $\begin{array}{c}\text { Contralateral tumor } \\
\mathrm{n}(\%)\end{array}$ & $p$ value \\
\hline Median age, years (min-max), mean & $59.50(39-81), 60.30$ & $59.50(39-81), 60.30$ & $>0.05$ \\
\hline$\leq 40$ & $1(10)$ & $1(10)$ & \\
\hline $41-50$ & $2(20)$ & $2(20)$ & \\
\hline $51-60$ & $2(20)$ & $2(20)$ & \\
\hline$>60$ & $5(50)$ & $5(50)$ & \\
\hline Menopausal status & & & $>0.05$ \\
\hline Premenopausal & $3(30)$ & $3(30)$ & \\
\hline Postmenopausal & $7(70)$ & $7(70)$ & \\
\hline Breast surgery & & & 0.684 \\
\hline Breast conserving surgery & $2(20)$ & $3(30)$ & \\
\hline Subcutaneous mastectomy (TSSM) & $1(10)$ & $1(10)$ & \\
\hline Mastectomy & $7(70)$ & $6(60)$ & \\
\hline Axillary surgery & & & $>0.05$ \\
\hline Partial axillar lymphadenectomy & $0(0)$ & $0(0)$ & \\
\hline Radical axillar lymphadenectomy & $10(100)$ & $10(100)$ & \\
\hline Tumor size $(\mathrm{mm})$, mean value $\pm \mathrm{SD}$; median; min-max & $\begin{array}{c}33.40 \pm 15.96 ; 27.5 \\
15-60\end{array}$ & $\begin{array}{c}17.40 \pm 10.76 ; 16.5 \\
2-35\end{array}$ & 0.011 \\
\hline Tumor size $(\mathrm{mm})$ groups & & & 0.035 \\
\hline$\leq 20 \mathrm{~mm}$ & $2(20)$ & $7(70)$ & \\
\hline$>20 \leq 50 \mathrm{~mm}$ & $6(60)$ & $3(30)$ & \\
\hline$>50 \mathrm{~mm}$ & $2(20)$ & $\mathrm{x}$ & \\
\hline Tumor focality & & & 0.315 \\
\hline Unifocal & $6(60)$ & $9(90)$ & \\
\hline Multifocal & $2(20)$ & $\mathrm{x}$ & \\
\hline Multicentric & $2(20)$ & $1(10)$ & \\
\hline Number of tumori foci & & & 0.280 \\
\hline Single focus & $6(60)$ & $9(90)$ & \\
\hline More than one focus & $4(40)$ & $1(10)$ & \\
\hline Histological type & & & 0.631 \\
\hline \multicolumn{4}{|l|}{ 1.IBC-NST, ILC special subtype } \\
\hline IDC & $7(70)$ & $6(60)$ & \\
\hline ILC & $3(30)$ & $3(30)$ & \\
\hline 2.mixed IBC-NST and special subtypes & $\mathrm{x}$ & $\mathrm{x}$ & \\
\hline \multicolumn{4}{|l|}{ 3.IBC-NST with focal specialized subtype } \\
\hline IDC + carcinoma with apocrine differentiation & $x$ & $1(10)$ & \\
\hline 4.Other special subtypes & $x$ & $\mathrm{x}$ & \\
\hline Nottingham grade & & & 0.743 \\
\hline Grade 1 & $3(30)$ & $3(30)$ & \\
\hline
\end{tabular}




\begin{tabular}{|c|c|c|c|}
\hline $\begin{array}{l}\text { Synchronous tumors, } n=10(100) \\
\text { Characteristics }\end{array}$ & $\begin{array}{c}\text { Dominant tumor } \\
\mathrm{n}(\%)\end{array}$ & $\begin{array}{c}\text { Contralateral tumor } \\
\mathrm{n}(\%)\end{array}$ & $p$ value \\
\hline Grade 2 & $5(50)$ & $5(50)$ & \\
\hline Grade 3 & $x$ & $1(10)$ & \\
\hline Unknown & $2(20)$ & $1(10)$ & \\
\hline Associated DCIS & & & 0.853 \\
\hline Low nuclear grade DCIS & $x$ & $1(10)$ & \\
\hline Intermediate nuclear grade DCIS & $2(20)$ & $1(10)$ & \\
\hline High nuclear grade DCIS & $1(10)$ & $x$ & \\
\hline None & $7(70)$ & $8(80)$ & \\
\hline Lymphovascular invasion & & & 0.739 \\
\hline Positive & $\mathrm{x}$ & $1(10)$ & \\
\hline Negative & $10(100)$ & $9(90)$ & \\
\hline Perineural invasion & & & $>0.05$ \\
\hline Positive & $2(20)$ & $2(20)$ & \\
\hline Negative & $8(80)$ & $8(80)$ & \\
\hline Margins & & & 0.739 \\
\hline Ro & $9(90)$ & $10(100)$ & \\
\hline $\mathrm{R} 1$ & $1(10)$ & $0(0)$ & \\
\hline Pathologic T & & & 0.035 \\
\hline pT1 & $2(20)$ & $7(70)$ & \\
\hline pT2 & $6(60)$ & $3(30)$ & \\
\hline pT3 & $2(20)$ & $3(30)$ & \\
\hline pT4 & $\mathrm{x}$ & $\mathrm{x}$ & \\
\hline Number invaded lymph nodes, mean (min-max), SD & $3.40(0-12), 4.0$ & $1.20(0-6), 1.98$ & 0.190 \\
\hline Nodal status (pN) & & & 0.143 \\
\hline pNO & $3(30)$ & $6(60)$ & \\
\hline $\mathrm{pN1}$ & $3(30)$ & $3(30)$ & \\
\hline $\mathrm{pN2}$ & $3(30)$ & $1(10)$ & \\
\hline $\mathrm{pN} 3$ & $1(10)$ & $\mathrm{x}$ & \\
\hline Anatomic stage (TNM) & & & 0.007 \\
\hline Stage IA & $\mathrm{x}$ & $4(40)$ & \\
\hline Stage IB & $\mathrm{x}$ & $\mathrm{x}$ & \\
\hline Stage IIA & $2(20)$ & $4(40)$ & \\
\hline Stage IIB & $4(40)$ & $1(10)$ & \\
\hline Stage IIIA & $3(30)$ & $1(10)$ & \\
\hline Stage IIIB & $\mathrm{x}$ & $\mathrm{x}$ & \\
\hline Stage IIIC & $1(10)$ & $x$ & \\
\hline Stage V & $x$ & $x$ & \\
\hline Smoker & $2(20)$ & & \\
\hline
\end{tabular}

$S D$ - standard deviation; IBC-NST - invasive breast carcinoma of no special type; IDC - invasive ductal carcinoma;

ILC - invasive lobular carcinoma; DCIS - ductal carcinoma in situ; RO-negative surgical margins; $R 1$ - positive surgical margins 
TABLE 3. Molecular pathology features in metachronous and synchronous tumors

\begin{tabular}{|c|c|c|c|c|c|c|}
\hline & \multicolumn{3}{|c|}{ Metachronous, $\mathrm{n}=\mathbf{2 5}(\mathbf{1 0 0 \% )}$} & \multicolumn{3}{|c|}{ Synchronous, $\mathrm{n}=10(100 \%)$} \\
\hline & 1st tumor & 2nd tumor & $p$ value & 1st tumor & 2nd tumor & $p$ value \\
\hline ER status & & & 0.370 & & & 0.710 \\
\hline Low positive & $1(4)$ & $\mathrm{x}$ & & $\mathrm{x}$ & $\mathrm{x}$ & \\
\hline Positive & $7(28)$ & $9(36)$ & & $6(60)$ & $5(50)$ & \\
\hline Negative & $12(48)$ & $7(28)$ & & $1(10)$ & $2(20)$ & \\
\hline Unknown & $4(16)$ & $9(36)$ & & $3(30)$ & $3(30)$ & \\
\hline Mixed, ER+, ER- & $1(4)$ & $\mathrm{x}$ & & $\mathrm{x}$ & $\mathrm{x}$ & \\
\hline PR status & & & 0.254 & & & $>0.05$ \\
\hline Positive & $5(20)$ & $7(28)$ & & $5(50)$ & $5(50)$ & \\
\hline Negative & $15(60)$ & $9(36)$ & & $2(20)$ & $2(20)$ & \\
\hline Unknown & $4(16)$ & $9(36)$ & & $3(30)$ & $3(30)$ & \\
\hline Mixed, PR+,PR- & $1(4)$ & $\mathrm{x}$ & & $\mathrm{x}$ & $\mathrm{x}$ & \\
\hline HER2 status & & & 0.964 & & & $>0.05$ \\
\hline Positive & $2(8)$ & $1(4)$ & & $1(10)$ & $1(10)$ & \\
\hline Negative & $18(72)$ & $15(60)$ & & $6(60)$ & $6(60)$ & \\
\hline Unknown & $4(16)$ & $9(36)$ & & $3(30)$ & $3(30)$ & \\
\hline Mixed, HER2-,- & $1(4)$ & $\mathrm{x}$ & & $\mathrm{x}$ & $\mathrm{x}$ & \\
\hline Positive AR & $2(8)$ & $1(4)$ & 0.730 & $\mathrm{x}$ & $\mathrm{x}$ & $\mathrm{x}$ \\
\hline HR status & & & 0.387 & & & 0.456 \\
\hline $\mathrm{ER}+, \mathrm{PR}-$ & $3(12)$ & $2(8)$ & & $1(10)$ & $5(50)$ & \\
\hline $\mathrm{ER}+, \mathrm{PR}+$ & $5(20)$ & $7(28)$ & & $5(50)$ & $\mathrm{x}$ & \\
\hline ER-, PR+ & $\mathrm{x}$ & $\mathrm{x}$ & & $\mathrm{x}$ & $x$ & \\
\hline ER-, PR- & $12(48)$ & $7(28)$ & & $1(10)$ & $2(20)$ & \\
\hline Unknown & $4(16)$ & $9(36)$ & & $3(30)$ & $3(30)$ & \\
\hline Mixed $\mathrm{T}^{1}$ & $1(4)$ & $x$ & & $x$ & $x$ & \\
\hline \multicolumn{2}{|c|}{ Biomarker-defined subtypes } & & 0.217 & & & 0.620 \\
\hline $\mathrm{ER}+, \mathrm{HER} 2-$ & $7(28)$ & $9(36)$ & & $5(50)$ & $4(40)$ & \\
\hline ER+, HER2+ & $1(4)$ & $x$ & & $1(10)$ & $1(10)$ & \\
\hline ER-, HER2+ & $1(4)$ & $1(4)$ & & $\mathrm{x}$ & $x$ & \\
\hline ER-, HER2- & $11(44)$ & $6(24)$ & & $1(10)$ & $2(20)$ & \\
\hline Unknown & $4(16)$ & $9(36)$ & & $3(30)$ & $3(30)$ & \\
\hline Mixed $\mathrm{T}^{2}$ & $1(4)$ & $\mathrm{x}$ & & $x$ & $\mathrm{x}$ & \\
\hline Ki-67 & & & 0.651 & & & $>0.05$ \\
\hline$<14 \%$ & $4(16)$ & $3(12)$ & & $1(10)$ & $1(10)$ & \\
\hline$\geq 14 \%$ & $7(28)$ & $9(36)$ & & $6(60)$ & $6(60)$ & \\
\hline Unknown & $14(56)$ & $13(52)$ & & $3(30)$ & $3(30)$ & \\
\hline Mixed $\mathrm{T}, \geq 14 \%^{3}$ & $1(4)$ & $x$ & & $x$ & $x$ & \\
\hline Intrinsec subtype & & & 0.111 & & & 0.902 \\
\hline Luminal A & $\mathrm{x}$ & $2(8)$ & & $x$ & $1(10)$ & \\
\hline Luminal B, HER2- & $5(20)$ & $6(24)$ & & $5(50)$ & $3(30)$ & \\
\hline Luminal B, HER2+ & $1(4)$ & $x$ & & $1(10)$ & $1(10)$ & \\
\hline HER2+ & $1(4)$ & $1(4)$ & & $\mathrm{x}$ & $\mathrm{x}$ & \\
\hline TNBC & $11(44)$ & $6(24)$ & & $1(10)$ & $2(20)$ & \\
\hline Unknown & $6(24)$ & $10(40)$ & & $3(30)$ & $3(30)$ & \\
\hline Mixed $\mathrm{T}^{4}$ & $1(4)$ & $x$ & & $x$ & $x$ & \\
\hline
\end{tabular}

ER - estrogen receptor; $P R$ - progesteron receptor; HER2 - human epidermal growth factor receptor type2; Ki-67 - marker of proliferation; $T N B C$ - triple negative breast cancer; Mixed $T^{1}$ - mixed tumor subtypes ER+,PR+ and ER-, PR-; Mixed $T^{2}-$ mixed tumor subtypes ER+, HER2-and ER-, HER2-; Mixed T, $\geq 14 \%{ }^{3}$ - mixed tumor molecular subtypes, both having Ki-67 $\geq 14 \%$; Mixed $T^{4}$ - mixed tumor with mixed molecular subtypes Luminal B (HER2-) and TNBC. 
were further analyzed and compared between $\mathrm{mBBC}$ and SBBC (Table 4).

Regarding ER receptor status, a higher percentage of ER-discordant tumors ( $57.1 \%$ vs. $14.3 \%$ ) in the $\mathrm{mBBC}$ group than the $\mathrm{sBBC}$ group, however without statistical significance between groups $(p=0.118)$. There was a higher percentage of PR-discordant tumors $(71.4 \%, p=$ 0.008 ) in metachronous group than the synchronous, where was found no discordance and a higher percentage of positive concordant $(71.4 \%)$ tumors $(p=0.257)$, with a overall statistical significant difference between groups $(p<0.001)$. In the synchronous group was also found a higher rate of ER positive concordance of $71.4 \%(p=0.102)$ compared to the metachronous group $(28.6 \%)(p=0.118$, overall statistical significance). Concerning HER2 status, in both groups, were more often encountered negative concordant values (92.9\%, 85.7\%, $p=0.219)$, however the only significant statistical difference was observed in the $\mathrm{MBBC}$ group $(p=0.001)$. Compared to $m B B C$ group SBBC group was more likely to have concordant HR status $(85.7 \%$ vs. $28.6 \%, p=0.010)$, concordant ER,HER2 status ( $85.7 \%$ vs. $35.7 \%, p=0.024)$, with statistical significance, and concordant molecular subtype $(71.4 \%$ vs. $33.3 \%, p=$ $0.105)$, though without significant statistical difference.

With regard to systemic therapy, no statistically significant differences were observed between the first and second tumor in metachronous and synchronous groups or among groups. In the $\mathrm{mBBC}$ group, $76 \%$ of the patients received adjuvant chemotherapy for the first tumor and $52 \%$ patients for the second $(p=0.639)$ and $40 \%$ of the patients in the synchronous group ( $p=$ 0.208 , mBBC vs. sBBC patients). Adjuvant hormonotherapy was less prevalent in the metachronous group $(36 \%)$ than in synchronous patients $(60 \%)(p=101)$. HER2-directed treatment received one patient (10\%) in SBBC group, 2 patients (8\%) for their first tumor in metachronous group and 1 patient (4\%) for the second tumor $(p=0.876)$. Adjuvant radiotherapy was also likewise observed among groups ( $p=0.876 ; \mathrm{mBBC}, 76 \%$ index tumor, $48 \%$ second tumor; sBBC, $60 \%$ patients).

In terms of local recurrence and distant metastasis, there was no statistically significant difference between the two groups. It was encountered one case of primary metastatic disease (4\%) in the metachronous group. Loco-regional recurrence was observed in each group,

TABLE 4. Concordance of molecular pathology characteristics between first tumor and second tumor in bilateral breast cancer, within and between groups

\begin{tabular}{|c|c|c|c|c|c|}
\hline & MBBC ( $n=25$, valid $\%)$ & $p$ value & SBBC $(n=10$, valid $\%)$ & $p$ value & $p$ value \\
\hline ER status & & 0.135 & & 0.102 & 0.118 \\
\hline Both positive & $4(28.6)$ & & $5(71.4)$ & & \\
\hline Both negative & $2(14.3)$ & & $1(14.3)$ & & \\
\hline Discordant & $8(57.1)$ & & $1(14.3)$ & & \\
\hline PR status & & 0.008 & & 0.257 & $<0.001$ \\
\hline Both positive & $1(7.1)$ & & $5(71.4)$ & & \\
\hline Both negative & $3(21.4)$ & & $2(28.6)$ & & \\
\hline Discordant & $10(71.4)$ & & $0(0)$ & & \\
\hline HER2-status & & 0.001 & & 0.059 & 0.219 \\
\hline Both positive & $0(0)$ & & $1(14.3)$ & & \\
\hline Both negative & 13(92.9) & & $6(85.7)$ & & \\
\hline Discordant & $1(7.1)$ & & $0(0)$ & & \\
\hline HR status & & 0.109 & & 0.059 & 0.010 \\
\hline Concordant & $4(28.6)$ & & $6(85.7)$ & & \\
\hline Discordant & $10(71.4)$ & & $1(14.3)$ & & \\
\hline ER, HER2 status & & 0.285 & & 0.059 & 0.024 \\
\hline Concordant & $5(35.7)$ & & $6(85.7)$ & & \\
\hline Discordant & $9(64.3)$ & & $1(14.3)$ & & \\
\hline Ki-67 & & 0.197 & & 0.257 & 0.517 \\
\hline Both $<14 \%$ & $1(12.5)$ & & $0(0)$ & & \\
\hline Both $\geq 14 \%$ & $5(62.5)$ & & $5(71.4)$ & & \\
\hline Discordant & $2(25)$ & & $2(28.6)$ & & \\
\hline Molecular type & & 0.248 & & 0.257 & 0.105 \\
\hline Concordant & $4(33.3)$ & & $5(71.4)$ & & \\
\hline Discordant & $8(66.7)$ & & $2(28.6)$ & & \\
\hline
\end{tabular}

$m B B C$ - metachronous bilateral breast cancer; $s B B C$ - synchronous bilateral breast cancer; $E R$ - estrogen receptor; PR - progesteron receptor; HER2 - human epidermal growth factor receptor type2; Ki-67-marker of proliferation 
2 cases in $\mathrm{mBCC}$ and 2 cases in SBBC, respectively. In the metachronous group, both cases of loco-regional relapse occured in the breast and in the $\triangle B B C$, one case in the breast and one in the axilla ( $p=0.577)$. Distant relapse occured in 16\% metachronous patients and $30 \%$ synchronous $(p=0.154)$. For both groups prevailed multiple metastatic sites (12\% mBBC; $20 \%$ sBBC; $p=0.137)$. In the metachronous group were found 4 cases $(16 \%)$ of distant recurrence, corresponding to one case that relapsed in the bone only (M1 bone) associated with concordant luminal B (HER2-negative) subtype, 1 case of relapse (M1) in the bone, lung and lymph node associated with discordant luminal B (HER2 negative) and TNBC subtypes and 2 cases related to concordant TNBC subtype which relapsed (M1) in bone and lung, respectively $\mathrm{M} 1$ bone and brain. In the synchronous group were observed 3 cases (30\%) of distant recurrence, representing one case of $\mathrm{M} 1$ lung related to a concordant luminal B (HER2 positive) subtype, another of M1 bone, lung and liver related to concordant luminal B (HER2 negative) and one case with M1 bone and lung relapse associated with discordant luminal $B$ (HER2 negativ) and luminal $A$. None of the relapsed cases had positive resection margins (R1). Both patients in $\mathrm{mBBC}$ group had loco-regional reccurence of the index tumor before developing carcinoma in the contralateral breast.

Concerning survival analysis, the metachronous and synchronous groups were compared in terms of overall survival, disease-free survival, loco-regional-recurrence free survival and distant-recurrence-free surviv- al. The follow-up period for $\mathrm{mBBC}$ group had a a mean of 136.92 months (range 17 - 528 months) and for sBBC a mean of 19.60 months (range 1-60 months). Regarding both groups, after a mean follow-up of 103.4 (range 1-528; median 51) months, there was observed one case of breast cancer related death, which occured in the synchronous group, after 20 months; due to the fact that all cases from the $\mathrm{mBBC}$ group were censored, no statistics could be computed for overall survival (means and medians). On the other hand, the statistical comparisons for OS were found to be statistically significant between groups, at all compared factor levels ( $p=0.005$, Log Rank, Breslow and Tarone-Ware).

Disease-free survival was 391.13 months estimated mean for metachronous tumors and 39.6 months for synchronous tumors and the difference between these groups was statistically significant at all compared levels ( $p=0.011$, Log Rank; $p=0.044$ Breslow; $p=0.024$ Tarone-Ware).

Loco-regional relapse-free survival was statistically significant later in the time course ( $p=0.027$, Log Rank) with no differences in the earlier and middle time course ( $p=0.088$, Breslow, $p=0.054$ Tarone-Ware). The estimated mean for metachronous was 486.31 months and for synchronous was 48.0 months.

Distant relapse-free survival estimated mean was found as 409.39 months for $\mathrm{mBBC}$ and 40.20 months for sBBC with a difference between groups of statistically significant value ( $p=0.003, \log$ Rank; $p=0.015$ Breslow; $p=0.007$ Tarone-Ware).

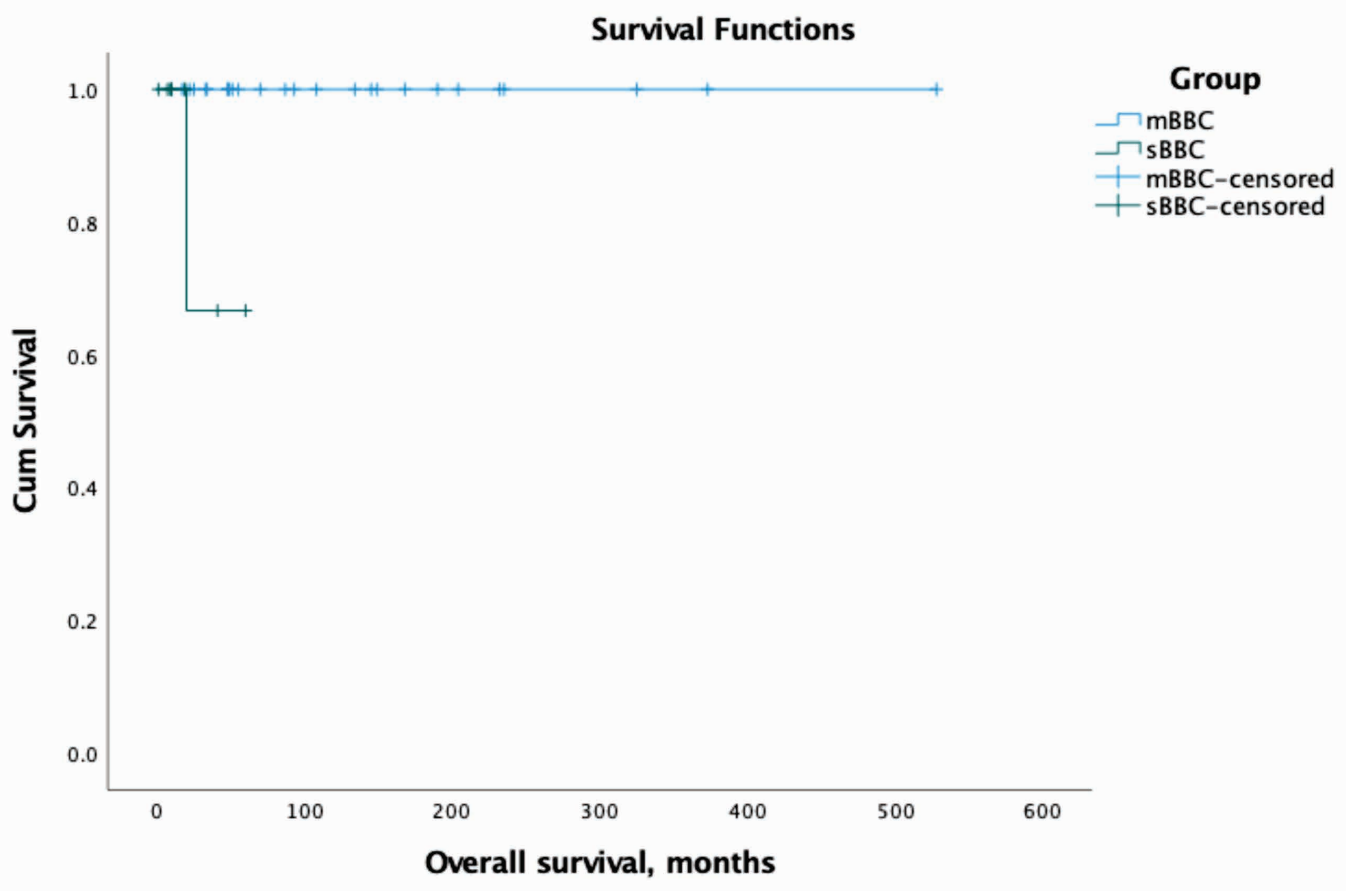

FIGURE 1.A. Overall survival. mBBC-metachronous bilateral breast cancer; sBBC-synchronous bilateral breast cancer 


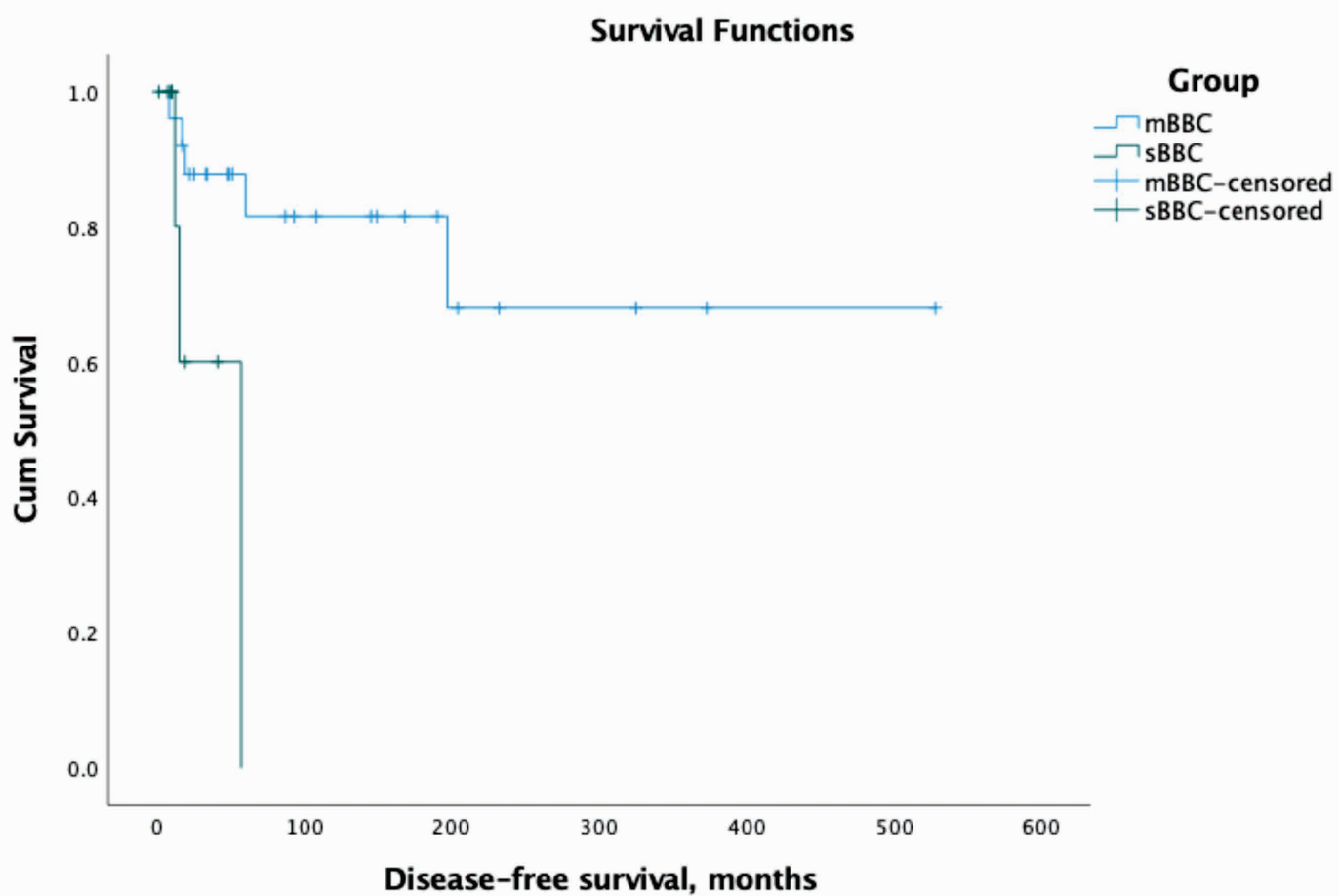

FIGURE 1.B. Disease-free survival. $m B B C$ - metachronous bilateral breast cancer; $S B B C$ - synchronous bilateral breast cancer

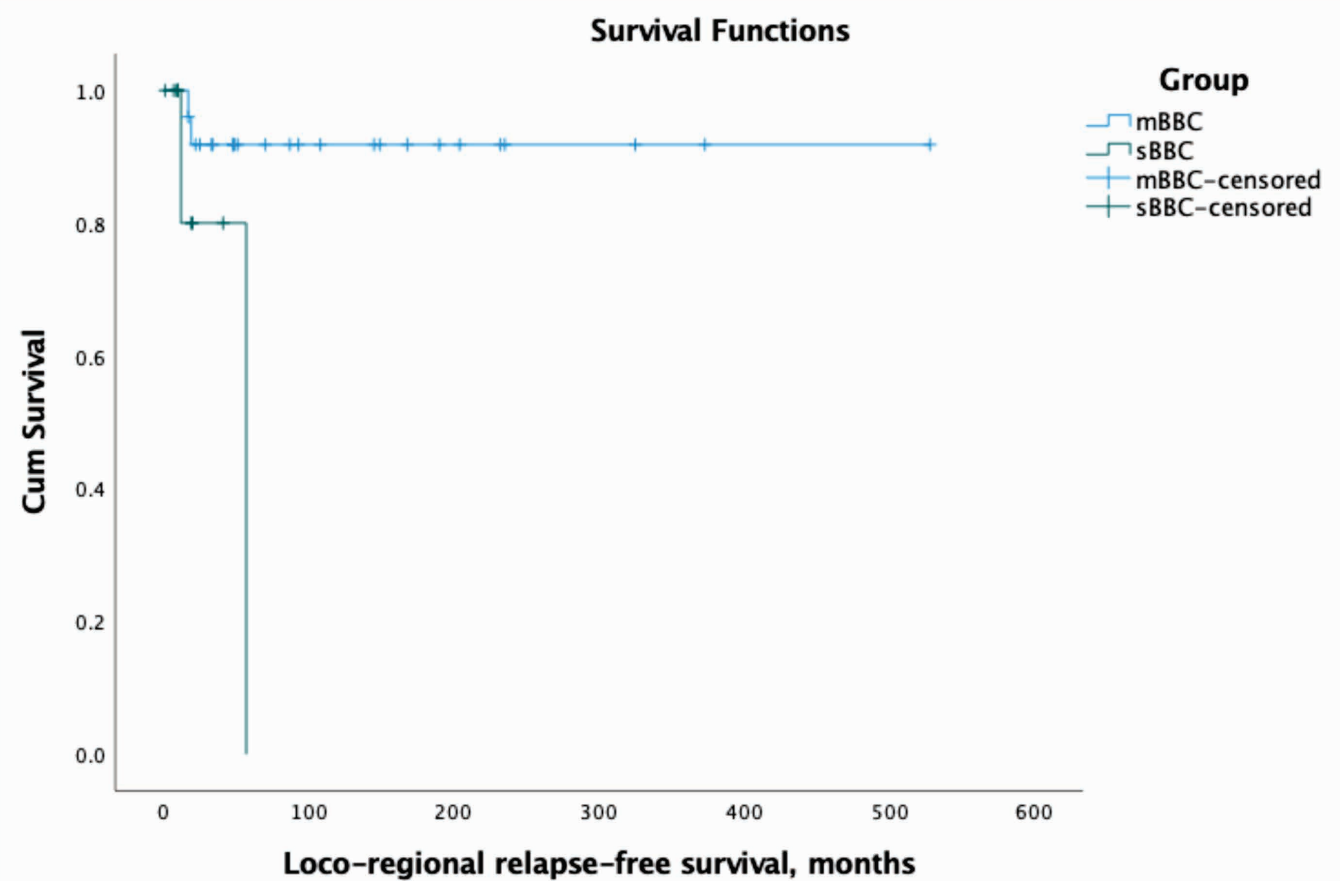

FIGURE 1.C. Loco-regional relapse-free survival. mBBC-metachronous bilateral breast cancer; sBBCsynchronous bilateral breast cancer

When comparing the patients from the metachronous group by means of time elapsed untill diagnosis of the second tumor, that is $<5$ years versus $>5$ years, it was observed that the time interval between $\mathrm{mBBC}$ tu- mors was no predictive of DFS ( $p=0.104$, Log Rank) or loco-regional RFS ( $p=0.904$, Log Rank), however it was statistically significant in terms of distant relapse-free survival (the mean distant relapse-free survival for pa- 


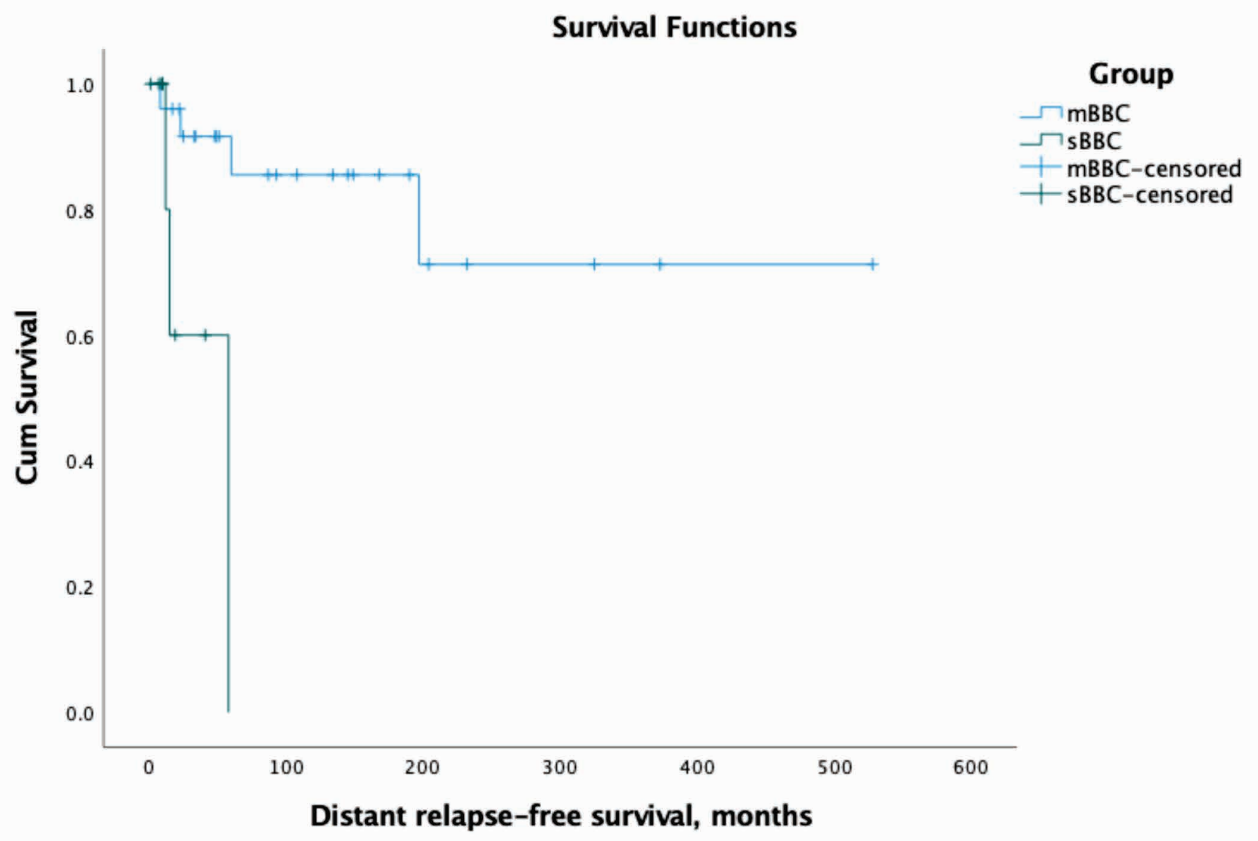

FIGURE 1.D. Distant relapse-free survival. mBBC - metachronous bilateral breast cancer; sBBCsynchronous bilateral breast cancer

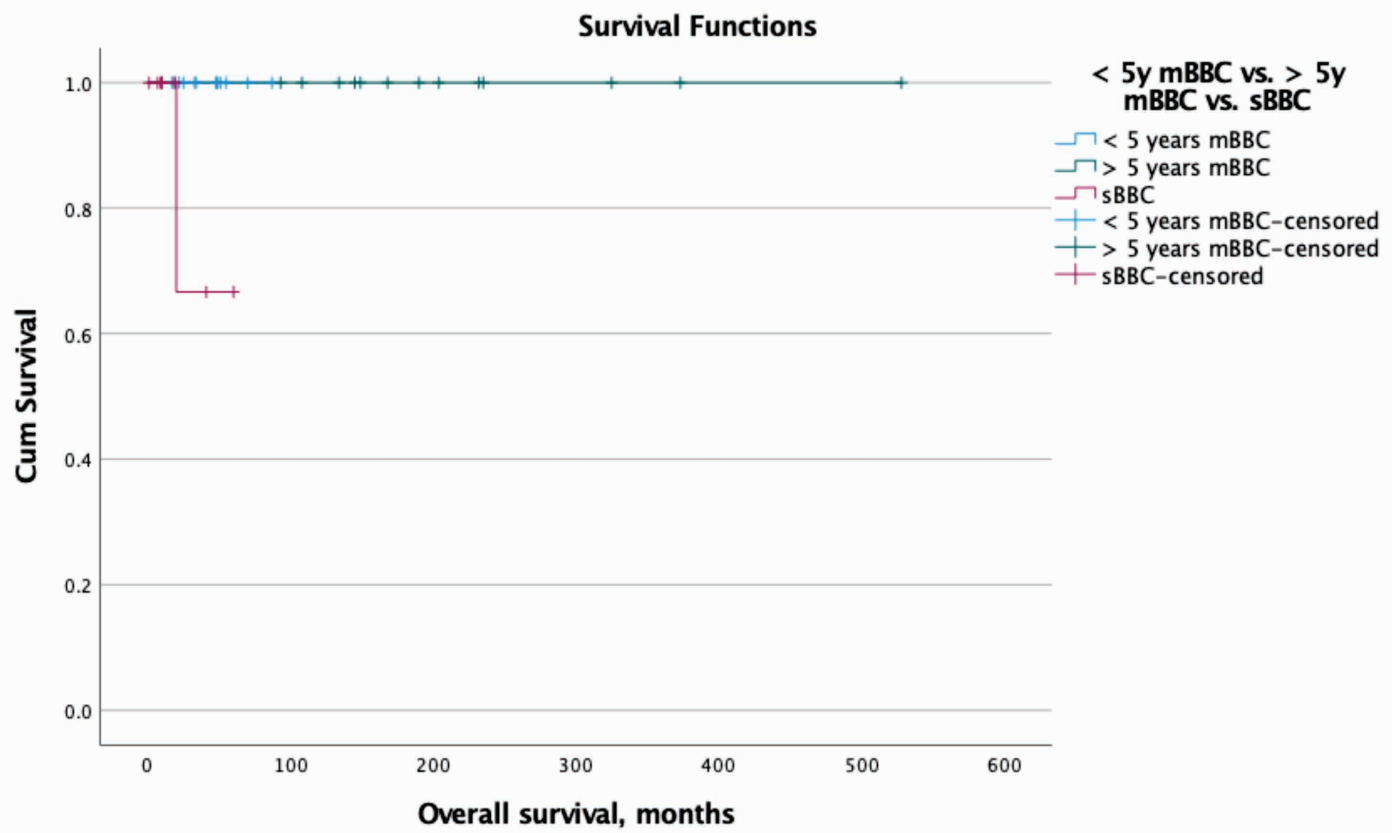

FIGURE 2.A. Overall survival. $m B B C$ - metachronous bilateral breast cancer; $s B B C$-synchronous bilateral breast cancer 


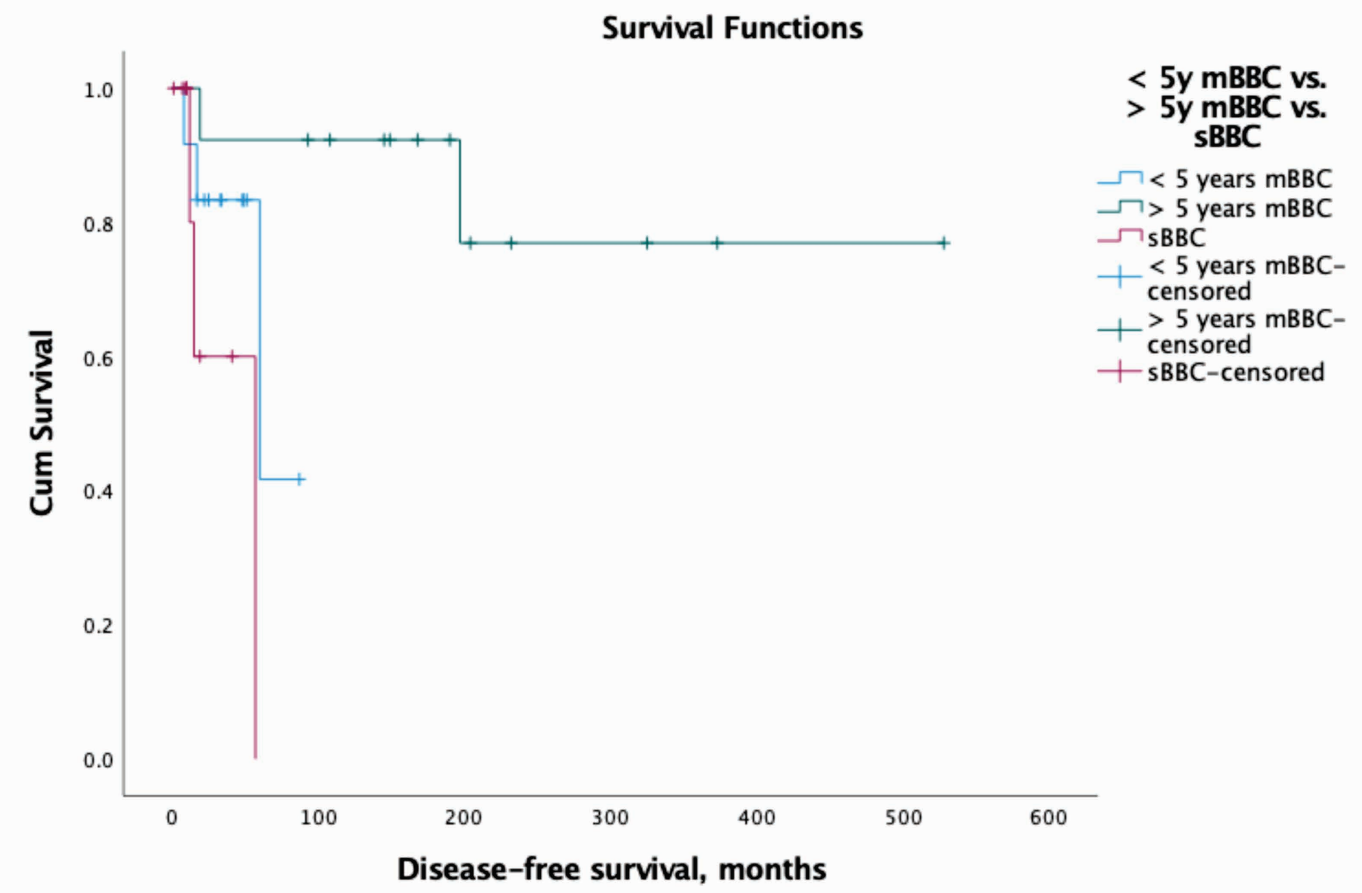

FIGURE 2.B. Disease-free survival. mBBC - metachronous bilateral breast cancer; sBBC - synchronous bilateral breast cancer

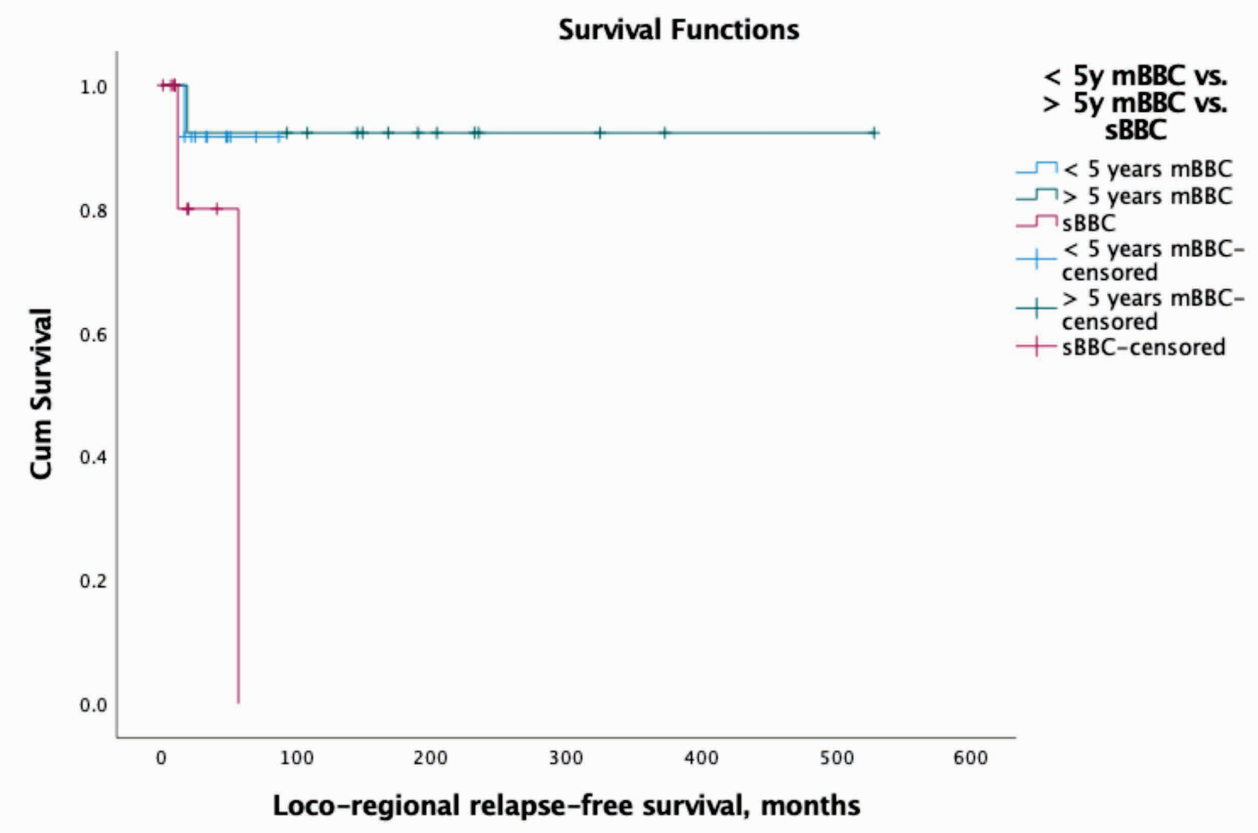

FIGURE 2.C. Loco-regional relapse-free survival. $m B B C$ - metachronous bilateral breast cancer; SBBC - synchronous bilateral breast cancer 


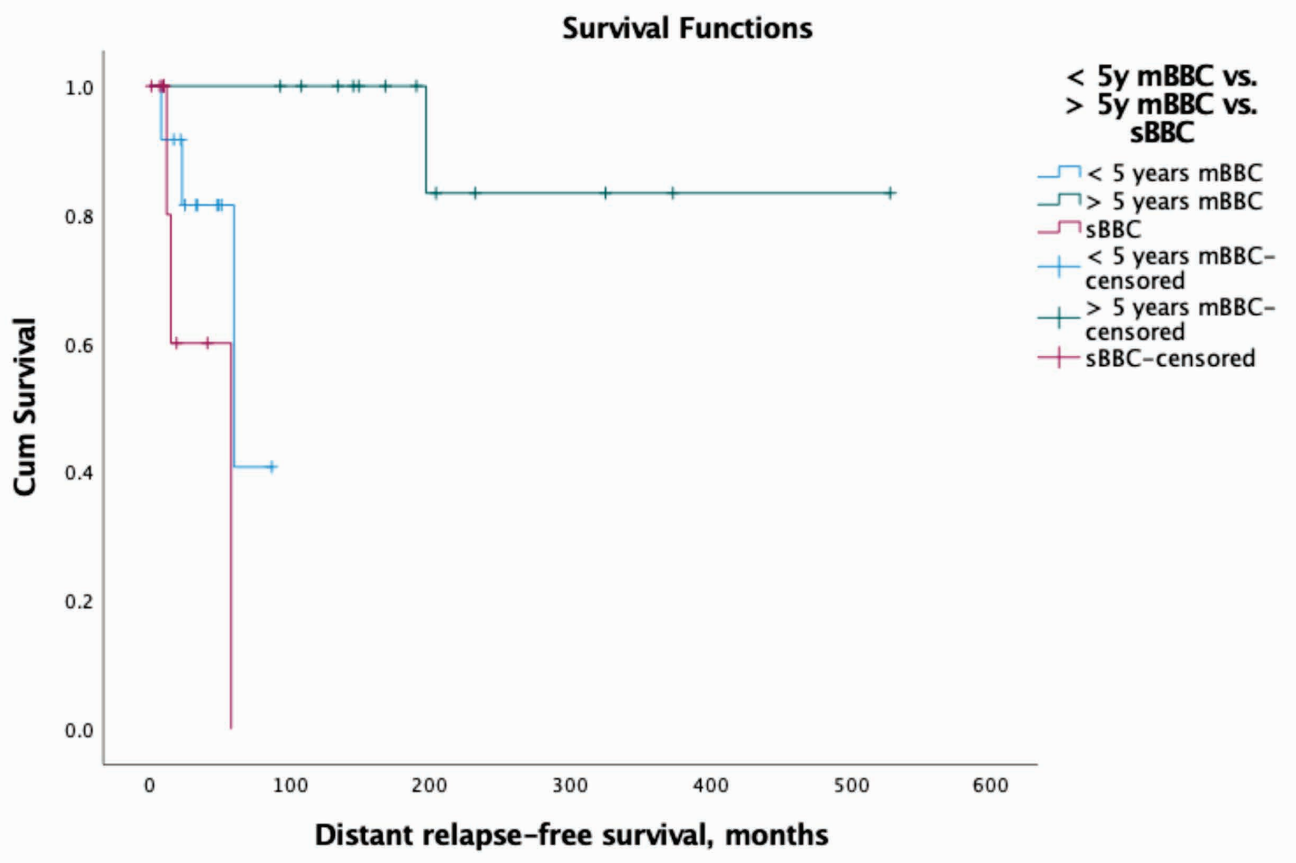

FIGURE 2.D. Distant relapse-free survival. $m B B C$ - metachronous bilateral breast cancer; $S B B C$ - synchronous bilateral breast cancer

tients with less than 5 years was 62.89 months, whereas it was 472.83 months for those with more than 5 years; $p=0.011$, Log Rank; $p=0.024$, Breslow; $p=$ 0.017 , Tarone Ware). Statistics and test of equality for overall survival could not be computed because all cases were censored.

We questioned if there was a difference in terms of survival analysis among the mBBC subgroups (time of diagnosis $<5$ years, $>5$ years) and sBBC. It was observed a statistical overall survival difference among groups ( $p$ $=0.018$ ). We found that SBBC patients had the worst prognosis based on disease-free survival rates and that $\mathrm{mBBC}$ patients diagnosed $<5$ years might be similar to sBBC (the mean disease-free survival for patients $<5$ years was 63.3 months and for SBBC it was 39.6 months, whereas for patients with more than 5 years was 437.9 months, $p=0.012$, Log Rank). There no statistical difference in terms of loco-regional relapse-free survival $(p=0.081$ Log Rank; $p=0.224$, Breslow; $p=0.148$ Tarone-Ware), although sBBC showed more frequent rates of loco-regional relapse and earlier in the time course. More frequent rates of distant metastasis were in the $\mathrm{sBBC}$ and $<5$ years $\mathrm{mBBC}$ with a statistically significant difference $(<5$ years $\mathrm{mBBC}$, mean 62.89 months; $>5$ years mBBC, 472.83 months; $\mathrm{sBBC}, 40.2$ months; $p=$ 0.001, Log Rank; $p=0.010$, Breslow, $p=0.004$ Tarone-Ware).

It was also analyzed the association of HR concordance, within tumor pairs, biomarker defined subtype (ER,HER2) concordance and molecular subtype concordance and disease-free survival. There was no sta- tistically significant difference between concordant $\mathrm{HR}$ group and discordant HR group in overall patients $(p=$ 0.280 , Log Rank), mBBC group ( $p=0.846$ ) or sBBC group $(p=0.445)$. Discordant biomarker defined subgroup patients were associated with better disease-free survival in overall patients ( $p=0.047, \log$ Rank) with no differences neither among patients in $\mathrm{mBBC}$ group $(p=$ 0.222 , Log Rank) nor sBBC ( $p=0.445$, Log Rank); in overall patients, distant relapse-free survival was also found to be significant, $p=0.015$, discordant ER,HER2 subgroups showed a better distant relapse-free survival, no statistical significance in terms of loco-regional relapse-free survivial $(p=0.199)$. Concordance in molecular subtype was associated with no statistically significant difference among groups $(p=0.221)$ or with groups (mBBC, $p=0.263 ; \mathrm{sBBC}, \mathrm{p}=0.592$ ).

\section{DISCUSSION}

Women with previous breast carcinoma are at increased risk for developing second primary breast cancer in the contralateral breast, Soerjomataram et al. reported a relative risk of $3.5 \%(7,28)$.

By Gollamudi et al., synchronous breast tumors were defined as development of cancer within 1 month of initial diagnosis, by Hartman et al. within 3 months and by Ibrahim, Chen et al. within 12 months of initial diagnosis $(12,20,29-33)$. In this study, was used the interval time of 3 months.

In the present study, out of the 658 evaluated breast carcinoma cases, 35 cases were diagnosed with bilater- 
al breast cancer. Several studies had reported the incidence of primary breast cancer development in the contralateral breast cancer as ranging from $1.4 \%$ to $11.8 \%$. (5,12,15-17,25-26). In our study, BBC incidence accounted for $5.3 \%$ of all cases. According to the existing literature, the incidence rate of metachronous breast cancer ranges from $0.3 \%$ to $1 \%$, whereas in this study was found a rate of $3.8 \%(12,27)$. The incidence rate of synchronous breast cancer was reported to be less than $2 \%(10,12)$. In this study, the incidence rate for synchronous was found to be $1.5 \%$. Studies report that most $\mathrm{mBBC}$ cases occur 5 years after the initial diagnosis; the median time in our study was 5.5 years, however in accordance with the literature $(12,27)$.

In the present study there was no statistical significant difference between metachronous and synchronous groups in terms of age at the initial diagnosis $(p=$ 0.339). Among the patients with metachronous breast cancer, the median age at the time of initial diagnosis was 58 years, finding surpassing reports from the existing literature, i.e, the median age at initial diagnosis was found as 52 years by Ozturk et al, as 51 years by Diaz et al. and 46 years by Liang et al. $(12,31,34)$.

Tumor characteristic showed no differences between the $\mathrm{mBBC}$ and $\mathrm{sBBC}$ groups, conversely there were statistical differences within the groups in terms of tumor diameters between the first and second tumors ( $p=0.004$, respectively $p=0.011)$; the second tumor in both metachronous and synchronous groups showed smaller diameters compared to the first malignancy. Kheirelseid et al. calculated the median first tumor diameter as $20 \mathrm{~mm}$ (range,1-100) an the median second tumor diameter as $15 \mathrm{~mm}$ (range, 1-82) in metachronous tumors and the median dominant and contralateral tumor diameters were reported as $24 \mathrm{~mm}$ (range, 1-130) respectively $12 \mathrm{~mm}$ (range, 1-140); they found a statistically significant difference in the synchronous group $(12,32)$. Ozturk et al. reported the mean tumor diameter in the metachronous group, as $22.3 \pm 13 \mathrm{~mm}$ for the first tumor and $14.3 \pm 11.5 \mathrm{~mm}$ for the second tumor; in the syhcronous group the mean tumor diameter was calculated as $21.5 \pm 13.3 \mathrm{~mm}$ in the dominant tumor and $12 \pm 8.3 \mathrm{~mm}$ in the contralateral tumor; they also detected a significant statistical difference in terms of first and second tumor diameters within groups ( $p=0.008, p<0.001$, respectively (12).

The median time for occurence of the second tumor differed among previous publications. By Kheirelseid et al. was reported as 46.8 months, by Liang et al. as 67 months (range, 14-432) and by Ozturk et al as 96 months (range, 12-191) $(12,32,34)$. In this study, the median time of metachronous cancer occurrence was found as 66 months (range, 16-372).

In terms of histological type, some previous publications reported higher prevalence of invasive lobular carcinoma in SBBC patients and others found invasive ductal carcinoma as the most common type of tumor $(5,6,12,20,35,36)$. In this study, in both groups, the invasive ductal carcinoma-NOS (IBC-NST) type, whether in combination (mixed or focal) with other special subtype carcinomas or not, was the most frequent histological type encountered.

Previously published studies, reported a particulary high level of concordance among SBBC tumors compared to $\operatorname{mBBC}(5,31,37,38)$. Huber et al. confirmed a $90.7 \%$ level of concordance for HR and suggested that this observation reflects the common environment where $\mathrm{SBBC}$ tumors developed in contrast to $\mathrm{mBBC}$ tumors, that show a non-significant level of HR-concordance because of intercurrent factors, i.e adverse effects of treatments for the index tumor, such as chemotherapy, anti-hormone treatment or menopausal status (5). The present study is in accordance with the literature, it was found a $85.7 \%$ level of concordance for HR status in $\mathrm{SBBC}$ patients and $28.6 \%$ in $\mathrm{mBBC}$ patients, with statistical significant difference between groups $(p=$ 0.010).

In the study of Chen et al. the rates for bilateral mastectomy and breast conserving surgery, reconstruction and combined surgery were $81.1 \%, 4.4 \%$, $3.0 \%$ and $11.5 \%$ for patients with $\mathrm{mBBC}$ and $86.2 \%$, $6.4 \%, 3.7 \%$ and $3.7 \%$, respectively for patients with sBBC (33). In the study of O'Brien et al. lumpectomy vs. mastectomy was performed to $57 \%$ vs. $43 \%$ of dominant and $48 \%$ vs. $51 \%$ of contralateral breast in $\mathrm{mBBC}$ patients, respectively $34 \%$ vs. $66 \%$ of dominant breast and $33 \%$ vs. $67 \%$ to contralateral breast in the synchronous group; axillary lymph node dissection in $46 \%$ vs. $29 \%$ in $\mathrm{mBBC}$ group and $37 \%$ vs. $33 \%$ in $\mathrm{sBBC}$ group (39). In our study, in the metachronous groups, mastectomy was performed to $96 \%$ of dominant breast and to $88 \%$ of contralateral, whereas breast conserving surgery to $4 \%$ of dominant, respectively to $8 \%$ of contralateral; radical axillar lymphadenectomy accounted for the majority of axillar surgery for $\mathrm{mBBC}$ tumors. In the $\mathrm{SBBC}$ breast conserving surgery was performed at a higher rate, to $20 \%$ of patients in the dominant breast and $30 \%$ to contralateral, conservative mastectomy accounted for $10 \%$ in the first tumor and also in the second, however, non-conservative mastectomy was performed in most cases (70\% first tumor; $60 \%$ second tumor); radical axillar lymphadenectomy was performed in all cases, both dominant and contralateral breast.

The is no consensus in the literature regarding the outcome of bilateral breast cancer, most studies showing a survival difference between $\mathrm{mBBC}$ and $\mathrm{sBBC}$ $(5,9,36)$. The study performed by Huber et al. revealed no difference between metachronous and synchro- 
nous patients in terms of overall or relapse-free survival, instead it was observed a higher rate of loco-regional recurrence in the $\mathrm{MBBC}$ patients (5). In Ozturk et al. series, there was no statistically significant difference between the metachronous and synchronous group in terms of overall survival, local recurrence-free survival and distant metastasis-free survival, however metachronous group displayed better prognosis in terms of disease-free survival ( $p=0.041$ ) (12). In the present study, the statistical analysis showed a survival difference between the two groups. The study by Ozturk et al. also revealed that the median survival was poorer among patients in whom metachronous tumor occured within less than 5 years ( $p=0.001)(12)$. In this study was found that the time interval between mBBC tumors was statistically significant only terms of distant relapse-free survival $(p=0.011)$. When the two metachronous subgroups were compared with synchronous group, was observed a statistical significance in terms of overall survival, disease-free survival and distant relapse-free survival.

\section{CONCLUSIONS}

Bilateral breast cancer represents a reality with a frequency that ranges between $1.4 \%$ and $11.8 \%$ as reported in the existing literature. In the present study, the most frequent histological type encountered was invasive ductal carcinoma, the finding was consistent with many previous reports. In terms of survival analysis, patients with synchronous tumors showed a worse disease outcome and distant relapse-free survival might be similar among synchronous patients and patients in whom metachronous tumor was diagnosed within less than 5 years. Patients from the $\mathrm{sBBC}$ group showed a higher rate of $H R$ concordance wirh positive concordant ER and PR receptor. Survival analysis showed no significant statistical difference in terms of hormonal receptor concordance or molecular subtype concordance. On the other hand, patients with ER,HER2 discordant tumors, compared to those cu concordant ER,HER2 tumors, had a better disease outcome. Consequently, analysing disease outcomes of breast cancer patients at a molecular level deserves further clinical observation.

Conflict of interest: none declared Financial support: none declared

\section{REFERENCES}

1. Sung H, Ferlay J, Siegel RL et al. Global Cancer Statistics 2020: GLOBOCAN Estimates of Incidence and Mortality Worldwide for 36 Cancers in 185 Countries. CA Cancer J Clin. 2021;71(3):209-249.

2. International Agency for Research on Cancer. Estimated number of new cases in 2020, Romania, females, all ages. CancerToday-IARC GLOBOCAN;2020. Accessed August 1, 2021. Available at: https://gco.iarc.fr/today/online-analysis-pie?v $=2020 \&$ mode $=$ cancer $\&$ mode $\_$population $=$ continents\&population $=900 \&$ populations $=$ $642 \&$ key $=$ total $\&$ sex $=2 \&$ cancer $=39 \&$ type $=0 \&$ statistic $=5 \&$ prevalence $=$ $0 \&$ population_group $=0$ \&ages group $\% 5 \mathrm{~B} \% 5 \mathrm{D}=0$ \&ages_group $\% 5 \mathrm{~B} \% 5 \mathrm{D}=$ $17 \&$ nb_items $=7 \&$ group_cancer $=$ 1\&include_nmsc $=1$ \&include_nmsc_other $=$ 1\&half_pie $=0 \&$ donut $=0 \#$ collapsegroup-0-4.

3. International Agency for Research on Cancer. Estimated number of deaths in 2020, Romania, females, all ages. Cancer Today-IARC, GLOBOCAN; 2020. Accessed August 1, 2021. Available at: https://gco.iarc. fr/today/online-analysis-pie? $v=2020 \&$ mode $=$ cancer\&mode_population $=$ continents\&population $=900 \&$ populations $=$ $642 \&$ key $=$ total $\&$ sex $=2 \&$ cancer $=39 \&$ type $=1 \&$ statistic $=5 \&$ prevalence $=$ 0\&population_group $=0$ \&ages group $\% 5 \mathrm{~B} \% 5 \mathrm{D}=0$ \&ages_group $\% 5 \mathrm{~B} \% 5 \mathrm{D}=$ 17\&nb_items $=7 \&$ group_cancer $=$ 1\&include $\_$nmsc $=1$ \&include $\_$nmsc_other $=$ 1\&half_pie $=0 \&$ donut $=0$ \#collapsegroup-0-4.

4. Centrul Național de evaluare și promovare a stării de sănătate. Analiza de situație;2019. Accessed August 2, 2021. Available at: https://insp.gov.ro/sites/cnepss/wp-content/ uploads/2019/10/Analiza-de-situatie.pdf.

5. Huber A, Seidler SJ, Huber DE. Clinicopathological Characteristics, Treatment and Outcome of 123 Patients with Synchronous or Metachronous Bilateral Breast Cancer in a Swiss Institutional Retrospective Series. Eur J Breast Health. 2020;16(2):129-136.

6. Eliyatkin N, Zengel B, Yagci A, et al. Properties of Synchronous Versus Metachronous Bilateral Breast Carcinoma with Long Time Follow Up. APJCP. 2015;16(12):4921-4926.

7. Newman LA, Sahin AA, Cunningham JE, et al. A case-control study of unilateral and bilateral breast carcinoma patients. Cancer. 2001;91(10):1845-1853.

8. Vuoto HD, García AM, Candás GB, et al. Bilateral breast carcinoma: clinical characteristics and its impact on survival. Breast J. 2010;16:625-632.

9. Hartman M, Czene K, Reilly M, et al. Incidence and prognosis of synchronous and metachronous bilateral breast cancer. J Clin Oncol. 2007:25:4210-4216.
10. Irvine T, Allen DS, Gillett $C$, et al. Prognosis of synchronous bilateral breast cancer. $\mathrm{Br} \mathrm{J}$ Surg. 2009;96:376-380.

11. Chen SF, Du CW, Yang P, et al. The molecular and clinicopathologiv characteristics of bilateral breast cancer. Sci Rep. 2013;3:2590.

12. Ozturk A, Alco G, Sarsenov D, et al. Synchronous and metachronous bilateral breast cancer: A long-term experience. JBUON. 2018;23(6):1591-1600.

13. Ding S, Sun X, Lu S, et al. Association of molecular subtype concordance and survival outcome in synchronous and metachronous bilateral breast cancer. The Breast. 2021;57:71-79.

14. Verkooijen HM, Chatelain V, Fioretta G, et al. Survival after bilateral breast cancer: results from a population based study. Breast Canc Res Treat. 2007; 105(3):347-357.

15. Pan B, Xu Y, Zhou Y, et al. The prognostic comparison among unilateral, bilateral, synchronous and metachronous bilateral breast cancer. A meta-analysis of studies from recent decade (2008-2018). Cancer Med. 2019;8:2908-2918.

16. Chen Y, Thompson W, Semenciw R, et al. Epidemiology of contralateral breast cancer. Cancer Epidemiol Biomark Prev. 1999;8(10):855-861.

17. Donovan AJ. Bilateral breast cancer. Surg Clin N Am. 1990;70:1141-1149. 
18. Parks RM, Derks MGM, Bastiaannet E, et al. Breast Cancer Epidemiology. In: Wyld Lynda, Markopoulos C, Leidenius M, et al. Breast Cancer Management for Surgeons. 1st ed. Cham: Springer International Publishing AG; 2018:19-29.

19. Jobsen JJ, van der Palen J, Ong F, et al. Bilateral breast cancer, synchronous and metachronous; differences and outcome. Breast Cancer Res Treat. 2015; 153:277-283.

20. Ibrahim NY, Sroor MY, Darwish DO. Impact of bilateral breast cancer on prognosis: synchronous versus metachronous tumors. Asian Pac J Cancer Prev. 2015; 16:1007-1010.

21. Takahashi H, Watanabe K, Takahashi M, et al. The impact of bilateral breast cancer on the prognosis of breast cancer: a comparative study with unilateral breast cancer. Breast Cancer. 2005;12:196-202.

22. Beckmann KR, Buckingham J, Craft $P$, et al. Clinical characteristics and outcomes of bilateral breast cancer in an Australian cohort. Breast. 2011;20:158-164.

23. Holm M, Tjønneland A, Balslev E, et al. Prognosis of synchronous bilateral breast cancer: a review and meta-analysis of observational studies. Breast Cancer Res Treat. 2014;146:461-475.

24. Jobsen JJ, van der Palen J, Ong F, et al. Synchronous, bilateral breast cancer: prognostic value and incidence. Breast. 2003;12:83-8.

25. Gogas J, Markopoulos C, Skandalakis P, et al. Bilateral breast cancer. Am Surgery. 1993:59:733-735.
26. Chen $Y$, Thompson W, Semenciw R, et al. Epidemiology of contralateral breast cancer. Cancer Epidemiol Biomark Prev. 1999;8:855-861.

27. Healey EA, Cook EF, Oraj EJ, et al. Contralateral breast cancer: clinical characteristics and impact on prognosis. J Clin Oncol. 1993;11:1545-1552.

28. Soerjomataram I, Louwman WJ, Lemmens VE, et al. Risks of second primary breast and urogenital cancer following female breast cancer in the south of The Netherlands, 1972-2001. Eur J Cancer. 2005;41:2331-2337.

29. Hartman M, Czene K, Reilly M, et al. Incidence and prognosis of synchronous and metachronous bilateral breast cancer. J Clin Oncol. 2007;25:4210-4216.

30. Gollamudi SV, Gelman RS, Peiro G, et al Breast conserving therapy for stage I-II synchronous bilateral breast carcinoma. Cancer. 1997;79:1362-1369.

31. Díaz R, Munárriz B, Santaballa A, et al. Synchronous and metachronous bilateral breast cancer: a long term single-institution experience. Med Oncol. 2012;29:16-24.

32. Kheirelseid EA, Jumustafa $\mathrm{H}$, Miller $\mathrm{N}$, et al. Bilateral breast cancer: analysis of incidence, outcome, survival and disease characteristics. Breast Cancer Res Treat. 2011; 126:131-140.

33. Chen JJ, Huang NS, Xue JY, et al. Surgical management for early-stage bilateral breast cancer patients in China. PLoS One. 2015;10:e0122692.

34. Liang X, Li D, Geng W, et al. The prognosis of synchronous and metachronous bilateral breast cancer in Chinese patients. Tumor Biol. 2013;34:995-1004

35. Intra M, Rotmensz N, Viale G, et al. Clinicopathologic characteristics of 143 patients with synchronous bilateral invasive breast carcinomas treated in a single institution. Cancer. 2004;101:905-912.

36. Qui R, Zhao W, Yang J, et al. Comparative analysis of outcomes and clinicopathological characteristics of synchronous and metachronous contralateral breast cancer: A study of the SEER database. J Breast Cancer. 2019;22:297-310.

37. Renz DM, Böttcher J, Baltzer PAT, et al. The contralateral synchronous breast carcinoma: a comparison of histology, localization, and magnetic resonanace imaging characteristics with the primary index cancer. Breast Cancer Res Treat. 2010; 120:449-459.

38. Huo D, Melkonian S, Rathouz PJ, et al. Concordance in histological and biological parameters between first and second primary breast cancers. Cancer. 2011;117:907-915.

39. O'Brien JA, Ho A, Wright GP, et al. Breast conserving surgery in bilateral breast cancer. Ann Surg Oncol. 2015;22:3389-3396. 